\title{
CONSTRUÇÃO E VALIDAÇÃO DE CARTILHA EDUCATIVA PARA PROMOÇÃO DA ALIMENTAÇÃO SAUDÁVEL ENTRE PACIENTES DIABÉTICOS
}

\author{
Development and validation of an educational booklet for health eating promotion \\ among diabetic patients
}

\section{Construcción y validación de cartilla educativa para la promoción de la alimentación saludable de pacientes diabéticos}

\author{
Marília de Sousa Gonçalves iD \\ Hospital Universitário Walter Cantídio - HUWC - Fortaleza (CE) - Brasil \\ Universidade Federal do Ceará - UFC - Fortaleza (CE) - Brasil
}

\author{
Roberta Freitas Celedônio (iD \\ Hospital Universitário Walter Cantídio - HUWC - Fortaleza (CE) - Brasil \\ Universidade Federal do Ceará - UFC - Fortaleza (CE) - Brasil
}

\section{Mayra Bezerra Targino iD}

Hospital Universitário Walter Cantídio - HUWC - Fortaleza (CE) - Brasil Universidade Federal do Ceará - UFC - Fortaleza (CE) - Brasil

\section{Ticiane de Oliveira Albuquerque iD}

Hospital Universitário Walter Cantídio - HUWC - Fortaleza (CE) - Brasil Universidade Federal do Ceará - UFC - Fortaleza (CE) - Brasil

\section{Pabyle Alves Flauzino iD}

Hospital Universitário Walter Cantídio - HUWC - Fortaleza (CE) - Brasil Universidade Federal do Ceará - UFC - Fortaleza (CE) - Brasil

\section{Alane Nogueira Bezerra iD \\ Centro Universitário FAMETRO - UNIFAMETRO - Fortaleza (CE) - Brasil Centro Universitário Christus - UNICHRISTUS - Fortaleza (CE) - Brasil Universidade Federal do Ceará - UFC - Fortaleza (CE) - Brasil}

\section{Natasha Vasconcelos Albuquerque iD}

Centro Universitário FAMETRO - UNIFAMETRO - Fortaleza (CE) - Brasil Hospital Universitário Walter Cantídio - HUWC - Fortaleza (CE) - Brasil

\section{Synara Cavalcante Lopes iD}

Hospital Universitário Walter Cantídio - HUWC - Fortaleza (CE) - Brasil

Universidade Federal do Ceará - UFC - Fortaleza (CE) - Brasil

\section{RESUMO}

Objetivo: Descrever o processo de construção e validação de conteúdo de uma cartilha educativa voltada para a orientação sobre alimentação saudável de pacientes com diabetes mellitus. Métodos: Trata-se de uma pesquisa metodológica com levantamento bibliográfico, elaboração e validação do material por especialistas no assunto. Os sete juízes possuíam especialização em diabetes e/ou com, no mínimo, dois anos de experiência na área, sendo duas enfermeiras, uma fisioterapeuta e quatro nutricionistas. Os itens avaliativos da cartilha consistiam em: objetivo, conteúdo, linguagem, relevância, ilustrações, layout, motivação e cultura, sendo cada item analisado separadamente através de escala tipo Likert. A análise da validação da tecnologia educativa foi realizada por meio da utilização do Índice de Validade de Conteúdo (IVC), com recomendação do ponto de corte superior a 0,78. Resultados: A cartilha educativa apresentou IVC global de 0,96. Apenas dois itens apresentaram valores de IVC abaixo do limite adotado. Os juízes realizaram observações referentes à aparência e ao conteúdo para o aperfeiçoamento da cartilha, as quais foram acatadas para a versão final. Conclusão: A cartilha educativa para orientação nutricional de pacientes com diabetes mellitus foi construída e validada em termos de aparência e conteúdo por juízes, devendo, assim, ser considerada para aperfeiçoar a adesão à terapia nutricional. Dessa forma, o material servirá de suporte aos profissionais e aos portadores de diabetes, para que superem as dificuldades que permeiam o autocuidado referente à alimentação para o controle da doença.

Descritores: Promoção da Saúde; Tecnologia Educacional; Estudos de Validação; Alimentação. 


\section{ABSTRACT}

Objective: To describe the process of construction and validation of the content of an educational booklet aimed at teaching about healthy eating for patients with diabetes mellitus. Methods: This is a methodological research with a bibliographical study that carried out the development and validation of the material by experts. The seven experts were specialized in diabetes and/or had at least two years of experience in the field: two nurses, one physiotherapist and four nutritionists. The booklet items evaluated were objective, content, language, relevance, illustrations, layout, and motivation and culture. Each item was analyzed separately using a Likert scale. The analysis of the validation of the educational technology was carried out using the Content Validity Index (CVI) with a recommended cutoff point higher than 0.78. Results: The educational booklet presented an overall CVI of 0.96. Only two items presented CVI values below the adopted limit. The experts recommended changes in the appearance and content for the refinement of the booklet that were included in the final version. Conclusion: The educational booklet for nutritional guidance of patients with diabetes mellitus was constructed and validated in terms of appearance and content by experts and should, therefore, be considered to improve adherence to nutritional therapy. Thus, the material will support professionals and patients with diabetes in overcoming the difficulties that permeate nutrition-related self-care for disease control.

Descriptors: Health Promotion; Educational Technology; Validation Studies; Feeding.

\section{RESUMEN}

Objetivo: Describir el proceso de construcción y validación de contenido de una cartilla educativa de orientación sobre la alimentación saludable de pacientes con diabetes mellitus. Métodos: Se trata de una investigación metodológica de búsqueda bibliográfica, elaboración y validación del material por especialistas en el tema. Los siete jueces tenían especialización en diabetes y/o con un mínimo de dos años de experiencia en el área: dos enfermeras, una fisioterapeuta y cuatro nutricionistas. Los ítems evaluativos de la cartilla eran: objetivo, contenido, lenguaje, relevancia, ilustraciones, layout, motivación y cultura y cada ítem ha sido analizado en separado a través de una escala tipo Likert. El análisis de la validación de la tecnología educativa ha sido realizada con la utilización del Índice de Validad de Contenido (IVC) con recomendación del punto de corte mayor que 0,78. Resultados: La cartilla educativa presentó IVC global de 0,96. Solamente dos ítems presentaron valores de IVC abajo del límite adoptado. Los jueces realizaron observaciones sobre la apariencia y el contenido para la mejoría de la cartilla las cuales fueron corregidas en la versión final. Conclusión: La cartilla educativa para la orientación nutricional de pacientes con diabetes mellitus ha sido construida y validada a través de la apariencia y el contenido por jueces y debe ser considerada para mejorar la adhesión de la terapia nutricional. De esa forma, el material va a servir de apoyo para los profesionales y los portadores de diabetes para que superen las dificultades que permean el autocuidado sobre la alimentación para el control de la enfermedad.

Descriptores: Promoción de la Salud; Tecnología Educacional; Estudios de Validación; Alimentación.

\section{INTRODUÇÃO}

A diabetes mellitus (DM) é definida como uma doença de caráter metabólico, caracterizada por hiperglicemia persistente decorrente da deficiência na produção de insulina ou na sua ação, ou em ambos os mecanismos. Representa um importante problema de saúde pública, devido à natureza crônica e suas complicações fortemente associadas ao tempo de exposição à hiperglicemia ${ }^{(1,2)}$.

No contexto das doenças crônicas, a DM merece destaque por sua elevada prevalência. A Federação Internacional de Diabetes (IDF) estimou que, em nível global, 425 milhões de adultos viviam com diabetes em 2017. Nesse mesmo ano, a DM foi responsável por 4 milhões de óbitos no mundo, de indivíduos entre 20 e 79 anos. De acordo com as projeções atuais, o número de pessoas com diabetes no mundo pode ultrapassar a marca de 628,6 milhões até $2045^{(2)}$. Assim, em consequência desse aumento, deve ser dada maior atenção aos cuidados relacionados com a prevenção de agravos e complicações da DM. Para tanto, é imprescindível que o indivíduo com diabetes mantenha o controle glicêmico adequado, o que exige mudanças no seu estilo de vida e grande aporte de cuidados ${ }^{(3)}$.

O crescimento da diabetes está associado a diversos fatores, dentre eles: padrão alimentar inadequado e sedentarismo ${ }^{(2)}$. Esses fatores de risco são comuns para as doenças crônicas não transmissíveis (DCNT), sendo temas prioritários da Política Nacional de Promoção da Saúde (PNPS)(4).

Considerando que o sucesso do tratamento das DCNT depende intimamente da participação e do envolvimento do usuário do serviço de saúde enquanto sujeito ativo de seu tratamento, a PNPS trouxe como um dos seus objetivos a promoção do empoderamento e da autonomia dos sujeitos e da coletividade através do desenvolvimento de habilidades pessoais e de competências em promoção e defesa da saúde e da vida ${ }^{(4,5)}$.

Um dos principais dispositivos para promover o cuidado da saúde é a educação em saúde, com um caráter mais ampliado, que auxilia não somente na prevenção de doenças como também no desenvolvimento da responsabilidade 
individual, sendo bastante utilizada para transformação de práticas e comportamentos individuais, além do desenvolvimento da autonomia e da qualidade de vida do indivíduo( ${ }^{(6)}$.

Associada ao emprego da educação em saúde, a educação nutricional vinculada ao plano alimentar permite ao indivíduo com diabetes uma melhor compreensão da influência dos alimentos na homeostase glicêmica e prevenção de agravos ${ }^{(1)}$. As orientações propostas podem influenciar no sucesso do tratamento da doença mediante parceria entre o profissional da saúde e o paciente, visando ao desenvolvimento da consciência para o autocuidado e à formação de atitudes, hábitos e práticas alimentares saudáveis ${ }^{(7)}$.

A utilização de estratégias eficazes para um melhor entendimento e motivação em serviços de saúde pode auxiliar a sanar a falta de conhecimento dos pacientes e de seus cuidadores a respeito da doença( ${ }^{(8)}$. O uso de materiais educativos impressos, como manuais, folhetos, folders, livretos, álbum seriado e cartilhas, são alternativas viáveis para informação e sensibilização da população( ${ }^{(9)}$. Esses recursos tornam-se muito úteis no controle da DM, pois facilitam a comunicação em saúde, a adesão ao tratamento, aprimoram o conhecimento e auxiliam no poder de decisão dos pacientes ${ }^{(10)}$.

A proposta da construção de um material educativo fundamenta-se no intuito de desenvolver ações de promoção da alimentação saudável como rotina no serviço de saúde e de fornecer aos profissionais um material de apoio para promover atividades de orientação alimentar e nutricional para pacientes diabéticos ${ }^{(11)}$. No entanto, para maximizar a efetividade da utilização de novos materiais escritos sobre educação em saúde, é necessário conhecer os procedimentos de abordagem para validar o conteúdo, buscando o desenvolvimento de instrumentos confiáveis e apropriados para determinada população ${ }^{(12)}$. Considerando a importância desses aspectos, o presente estudo teve como objetivo descrever o processo de construção e validação de conteúdo de uma cartilha educativa voltada para a orientação sobre alimentação saudável de pacientes com diabetes mellitus.

\section{MÉTODOS}

Trata-se de uma pesquisa com abordagem metodológica, em que a utilização sistemática dos conhecimentos existentes fomenta a elaboração, validação e avaliação de instrumentos e estratégias metodológicas ${ }^{(13)}$.

O estudo foi desenvolvido em três etapas: levantamento bibliográfico; elaboração das ilustrações, do layout, do design e dos textos, e validação aparente e de conteúdo do material educativo por especialistas ${ }^{(14)}$. No processo de construção da cartilha, para definição de assuntos relevantes a serem abordados na tecnologia educativa, realizou-se um levantamento bibliográfico no Guia Alimentar Para População Brasileira: Promovendo a Alimentação Saudável ${ }^{(15)}$, assim como no Manual de Nutrição da Sociedade Brasileira de Diabetes (SBD) ${ }^{(16)}$, nas Diretrizes da SBD 2017-2018 ${ }^{(1)}$ e nas Diretrizes de Cuidados Médicos em Diabetes da Associação Americana de Diabetes (ADA) ${ }^{(17)}$.

$\mathrm{Na}$ etapa 2, elaborou-se um roteiro de conteúdo a ser abordado, em uma sequência lógica, com sugestões de ilustrações que melhor representassem o conteúdo em tela. Enviaram-se as ilustrações a um profissional de ilustração e design para confeccionar os desenhos gráficos, formatar e diagramar. Submeteram-se o conteúdo preliminar e as ilustrações desenvolvidas ao processo de edição e diagramação, considerando os pressupostos para a construção e eficácia de materiais educativos, os quais estão relacionados com o conteúdo, linguagem, ilustração, layout e design ${ }^{(18)}$.

A etapa 3 constituiu-se do processo de validação do material construído por especialistas, dentre eles, 2 enfermeiras, 1 fisioterapeuta e 4 nutricionistas. Adotou-se, para a escolha dos participantes, a amostragem do tipo bola-de-neve ${ }^{(13)}$, sendo considerados especialistas da área de interesse conforme critérios adaptados de literatura consultada(19,20).

Para a realização da validação da tecnologia educativa desenvolvida, coletaram-se dados a partir de um instrumento adaptado da literatura ${ }^{(21)}$. A ferramenta consistia em duas partes: a primeira discorria sobre aspectos relacionados com a identificação do juiz, enquanto a segunda caracterizava-se por instruções relativas ao preenchimento do instrumento e os 41 itens avaliativos da cartilha, que correspondiam aos aspectos sobre objetivo, conteúdo, linguagem, relevância, ilustrações, layout, motivação e cultura. Ao final do instrumento, solicitou-se aos juízes que realizassem comentários gerais sobre a cartilha.

Realizou-se a análise da validação da tecnologia educativa por meio do Índice de Validade de Conteúdo (IVC), que mede a proporção da concordância sobre determinado assunto em um instrumento. Realizou-se a análise de cada item, separadamente, através de escala tipo Likert, com pontuações de 1 a 5 , em que: 1) discordo totalmente; 2 ) discordo; 3) não discordo nem concordo; 4) concordo; 5) concordo totalmente. As pontuações 4 e 5 foram consideradas como concordantes. Mais de seis especialistas avaliaram a cartilha e, nesse caso, a literatura recomenda ponto de 
corte superior a $0,78^{(22,23)}$. Dessa forma, para avaliação completa da cartilha educativa, utilizou-se o somatório de todos os IVC calculados separadamente, dividindo-os pelo número de itens do instrumento ${ }^{(13)}$.

A presente pesquisa foi submetida ao Comitê de Ética em Pesquisa da Universidade Federal do Ceará e aprovada sob o Parecer n. ${ }^{\circ}$ 2.357.269.

\section{RESULTADOS}

A cartilha educativa, denominada "Diabetes Mellitus: cartilha de nutrição", compôs-se, em sua versão final, por capa e 39 páginas, as quais abordavam os temas: Apresentação; DM (definição, classificação e complicações); Classificação Nutricional pelo Índice de Massa Corporal (IMC); Conhecendo os Nutrientes (carboidratos, fibras, proteínas e gorduras); A escolha dos alimentos (alimentos in natura ou minimamente processados, alimentos processados e ultraprocessados); Leitura dos rótulos de alimentos e informações nutricionais; Medidas caseiras; Higienização de frutas, verduras e legumes; Tipos de Adoçante; Hipoglicemia (conceito, sintomas e tratamento); Funcionamento intestinal; Uso de bebidas alcoólicas; Vida saudável; Fechamento da cartilha educativa.

Para a forma escrita, utilizaram-se tópicos com conteúdo explicativo. As ilustrações, realizadas por um design gráfico, estavam de acordo com a ideia de cada tópico abordado na tecnologia. Eram dispostas em cada página segundo o tema apresentado.

Após o processo de elaboração da cartilha educativa, procedeu-se à validação aparente e de conteúdo, realizada por sete especialistas, sendo: duas enfermeiras, uma fisioterapeuta e quatro nutricionistas. Seis concluíram a especialização em Diabetes na modalidade de Residência Multiprofissional em Saúde e uma atendia ao critério de experiência clínica na área. Encontrou-se como maior grau de titulação o mestrado, concluído por $28 \%$ ( $n=2)$ dos juízes.

A validação da tecnologia educativa deu-se através do cálculo do IVC, descrita na Tabela I. Na maioria dos itens, encontrou-se o IVC de, pelo menos, 0,86. Deve-se ressaltar que apenas dois itens, 3.5 e 4.5, relativos à escrita utilizada e à adequação do material a ser utilizado por qualquer profissional da área da saúde, apresentaram valores de IVC abaixo da concordância mínima adotada. No entanto, calculando-se a média do IVC para cada um dos aspectos avaliativos da cartilha, obteve-se um IVC superior a 0,90. Encontrou-se como média global do IVC o valor de 0,96, ratificando a validação da aparência e conteúdo junto aos especialistas (Tabela II).

Tabela I - Índice de validade de conteúdo para cada elemento do material educativo. Fortaleza, 2018.

\begin{tabular}{lcc}
\hline Itens da Cartilha Educativa & n & IVC \\
\hline Objetivos & $\mathbf{1 , 0}$ \\
\hline Os objetivos são coerentes com as necessidades de educação em saúde dos pacientes com diabetes mellitus (DM) & 7 & 1,0 \\
Esta tecnologia educativa é uma ferramenta que pode ser utilizada na educação em saúde do paciente com DM. & 7 & 1,0 \\
A tecnologia educativa é capaz de promover reflexão sobre os autocuidados no paciente diabético. & 7 & 1,0 \\
As informações contidas na tecnologia educativa promovem mudança de comportamento e atitude & $\mathbf{7}$ & 1,0 \\
\hline Conteúdo & $\mathbf{0 , 9 5}$ \\
\hline 2.1 A tecnologia educativa é apropriada para pacientes com DM. & 7 & 1,0 \\
2.2 A tecnologia educativa oferece informações sobre como realizar o autocuidado em pacientes diabéticos. & 7 & 1,0 \\
2.3 A tecnologia educativa ressalta a importância dos cuidados para prevenir o aparecimento ou agravamento complicações. & 7 & 1,0 \\
2.4 O texto está apresentado de forma clara e objetiva. & 6 & 0,86 \\
2.5 As informações apresentadas estão cientificamente corretas. & 7 & 1,0 \\
2.6 Os conteúdos são variados e suficientes para atingir os objetivos da tecnologia educativa. & 7 & 1,0 \\
2.7 Existe uma sequência lógica do conteúdo apresentado. & 6 & 0,86 \\
2.8 A divisão dos títulos e subtítulos do material são pertinentes. & 6 & 0,86 \\
2.9 As ideias chaves são pontos importantes e merecem destaque. & 7 & 1,0 \\
\hline Linguagem & $\mathbf{0 , 9 0}$ \\
\hline 3.1 As informações apresentadas são claras e compreensíveis. & 7 \\
3.2 O estilo de redação corresponde ao nível de conhecimento do público-alvo. & 1,0 \\
3.3 As informações estão bem estruturadas. & 6,86 \\
3.4 As informações estão em concordância com a ortografia. & 1,0 \\
3.5 A escrita utilizada é atrativa. & 6,86 \\
3.6 O título da tecnologia educativa é interessante e adequado. & 0,71 \\
\hline
\end{tabular}




\begin{tabular}{|c|c|c|}
\hline Relevância & & 0,95 \\
\hline 4.1 Os temas retratam pontos-chave que devem ser reforçados durante as consultas. & 7 & 1,0 \\
\hline 4.2 O material permite a transferência e generalizações do aprendizado a diferentes contextos. & 7 & 1,0 \\
\hline 4.3 A tecnologia educativa propõe ao aprendiz adquirir conhecimento para realizar o autocuidado. & 7 & 1,0 \\
\hline 4.4 A tecnologia educativa aborda os assuntos necessários para o paciente com DM. & 7 & 1,0 \\
\hline 4.5 A tecnologia educativa está adequada para ser usada por qualquer profissional da área da saúde. & 5 & 0,71 \\
\hline 4.6 O tema é atual e relevante. & 7 & 1,0 \\
\hline llustrações & & 0,94 \\
\hline 5.1 As ilustrações utilizadas são pertinentes com o conteúdo do material. & 7 & 1,0 \\
\hline 5.2 As ilustrações expressam a informação a ser transmitida. & 6 & 0,86 \\
\hline 5.3 O número de ilustrações está suficiente. & 7 & 1,0 \\
\hline 5.4 As legendas das ilustrações estão adequadas e auxilia o leitor a compreender a imagem. & 6 & 0,86 \\
\hline 5.5 Os personagens são carismáticos. & 7 & 1,0 \\
\hline Layout & & 0,98 \\
\hline 6.1 A apresentação da tecnologia educativa está atrativa. & 7 & 1,0 \\
\hline 6.2 A apresentação da tecnologia educativa está organizada de forma lógica. & 7 & 1,0 \\
\hline 6.3 O conteúdo está apresentado com letra em tamanho e fonte adequados para a leitura. & 7 & 1,0 \\
\hline 6.4 O tipo de letra utilizado facilita a leitura do material. & 6 & 0,86 \\
\hline 6.5 O contraste com cores diferentes foi feito de forma adequada. & 7 & 1,0 \\
\hline 6.6 A disposição do texto está adequada. & 7 & 1,0 \\
\hline 6.7 O número de páginas está adequado. & 7 & 1,0 \\
\hline Motivação & & 1,0 \\
\hline $7.1 \mathrm{O}$ título é atraente e desperta interesse para a leitura. & 7 & 1,0 \\
\hline 7.20 conteúdo desperta interesse para a leitura. & 7 & 1,0 \\
\hline 7.30 conteúdo está motivador e incentiva o leitor a prosseguir a leitura. & 7 & 1,0 \\
\hline Cultura & & 1,0 \\
\hline 8.1 O material está apropriado ao nível sociocultural do público-alvo proposto. & 7 & 1,0 \\
\hline Média global da tecnologia educativa 0,96 & & \\
\hline
\end{tabular}

Tabela II - Média do IVC em cada aspeto avaliativo da tecnologia educativa. Fortaleza, 2018.

\begin{tabular}{lc}
\hline Tema & Média \\
\hline 1- Objetivos & 1,0 \\
2- Conteúdo & 0,95 \\
3- Linguagem & 0,90 \\
4- Relevância & 0,95 \\
5- llustrações & 0,94 \\
6- Layout & 0,98 \\
7- Motivação & 1,0 \\
8- Cultura & 1,0 \\
Média global da tecnologia educativa & 0,96 \\
\hline
\end{tabular}

Analisaram-se as sugestões de cada profissional para o aperfeiçoamento da cartilha, acatando-as, tais como: reformulação de ilustração; substituição ou exclusão de termos técnicos; reformulação de formatação de texto; simplificação e reelaboração de frases, conforme constam no Quadro I.

Quadro I - Sugestões dos juízes sobre a aparência e o conteúdo da cartilha educativa. Fortaleza, 2018.

\begin{tabular}{|l|l|}
\hline Sugestões dos juízes & Modificações realizadas \\
\hline Reformulação de ilustração & $\begin{array}{l}\text { Reformulada figura da capa da cartilha educativa. Foi acrescentada à figura da } \\
\text { capa uma imagem que remete à ideia de alimentação e nutrição. }\end{array}$ \\
\hline Reformulação de formatação de texto & $\begin{array}{l}\text { Padronização de títulos em caixa alta e centralizados e em negrito. Aumento } \\
\text { do tamanho da fonte. }\end{array}$ \\
\hline Substituição/exclusão de termos técnicos & Substituído “lesão nervosa" por "lesão nos nervos". \\
\hline Simplificação e reformulação de frase & $\begin{array}{l}\text { Substituído "cicatrização difícil” por "difícil cicatrização". } \\
\text { Substituído "comprometendo a irrigação das extremidades" por "dificultando o } \\
\text { fluxo sanguíneo". } \\
\text { Substituído "você sabe os sintomas da hipoglicemia?" por "você conhece os } \\
\text { sintomas da hipoglicemia?" }\end{array}$ \\
\hline
\end{tabular}




\section{DISCUSSÃO}

No presente estudo, elaborou-se e validou-se um material educativo para a promoção da alimentação saudável entre pacientes com DM. O Ministério da Saúde alerta para fatores de riscos modificáveis, como a alimentação saudável, no enfretamento das doenças crônicas não transmissíveis no país. Além disso, recomenda a produção e distribuição de estratégias educativas que orientem e sensibilizem a população ${ }^{(4,24)}$. Visto isso, materiais abordando a temática supracitada, semelhante ao elaborado e validado pelo presente estudo, estão de acordo com as prioridades de saúde pública nacionais ${ }^{(4)}$.

Seguindo a tendência mundial, no Brasil, as DCNT estão em evidência, configurando um novo desafio para os gestores de saúde devido a seu forte impacto na morbimortalidade e na qualidade de vida dos indivíduos afetados ${ }^{(25)}$. A instituição de políticas, dentre elas, a Política Nacional de Prevenção do Diabetes e de Assistência Integral à Saúde da Pessoa Diabética (PLC 133/2017), que se encontra em tramitação, são necessárias para ampliar as atividades de prevenção e promover maior conhecimento sobre a DM, visto que se trata de uma enfermidade passível de controle mediante a combinação de tratamento, alimentação e estilo de vida ativo ${ }^{(26)}$.

Nesse contexto, as tecnologias educativas se mostram efetivas para promover saúde, pois aperfeiçoam o conhecimento e o enfrentamento do paciente, tornando-o capaz de entender como as próprias ações influenciam a sua condição de saúde ${ }^{(27)}$.

A cartilha educativa é uma proposta de trabalho para favorecer a educação nutricional de pessoas com diabetes e também um instrumento pelo qual se pode despertar a vontade do leitor na ampliação do seu potencial de autocuidado ${ }^{(28)}$. Sua divulgação para os usuários e os profissionais das unidades de saúde faria parte das estratégias de enfrentamento e controle da diabetes, dos problemas relacionados com a doença e de seus determinantes ${ }^{(26)}$.

No atual estudo, o processo de validação caracterizou-se pela multidisciplinaridade dos juízes, representando aspecto bastante favorável, visto que foi possível reunir diversos saberes especializados na temática abordada pelo material com observações pertinentes e complementares ${ }^{(29)}$. Outras pesquisas corroboram que o trabalho realizado por diferentes profissionais valoriza a equipe multiprofissional e possibilita diferentes concepções e enfoques sobre o mesmo tema ${ }^{(29-31)}$.

De maneira geral, as respostas dos juízes foram concordantes, como é possível visualizar nos resultados apresentados. Corroborando os dados apresentados, outros estudos metodológicos de desenvolvimento de tecnologias educativas também validaram seus materiais com altos índices estatísticos: a cartilha para alimentação saudável durante a gravidez foi validada com $0,91^{(11)}$; enquanto a construção de material para a prevenção de síndrome metabólica em adolescentes foi finalizada com IVC global de $0,98^{(29)}$.

Apesar de o IVC global ter demonstrado satisfação $(0,96)$, os juízes do atual estudo sugeriram mudanças relevantes para o aperfeiçoamento da cartilha educativa. Além disso, os juízes concordaram com a aplicabilidade do material educativo. Outros estudos que validaram tecnologias educativas impressas também utilizaram o IVC para validação de conteúdo e passaram por ajustes até a elaboração da versão final validada, enfatizando, dessa forma, a importância da realização dessa etapa para o desenvolvimento de um material qualificado ${ }^{(32,33)}$.

O presente estudo possui como limitação a não validação junto ao público-alvo. Tal fato impossibilita medir o entendimento e a efetividade do conteúdo contido na tecnologia educativa. Espera-se que as próximas etapas se configurem na aplicabilidade junto ao público-alvo, principalmente em ações voltadas para educação em saúde, para favorecer a otimização do cuidado por parte dos pacientes e profissionais e incentivar a promoção de hábitos alimentares saudáveis, prevenindo agravos e melhorando a qualidade de vida.

A cartilha resultante deste trabalho encontra-se disponível na íntegra e de forma gratuita no link: http://www2. ebserh.gov.br/documents/214604/0/Cartilha+Nutric\%CC\%A7a\%CC\%83o+e+Diabetes+compactado.pdf/c27a6bb7$7 \mathrm{bd0}-419 f-8 \mathrm{~cd} 1-5156255 \mathrm{cf} 518$

\section{CONCLUSÃO}

A cartilha educativa para orientação nutricional em DM, construída e validada em termos de aparência e conteúdo por juízes especializados, deve ser considerada no contexto de atividades educativas, com o intuito de aperfeiçoar a aderência à terapia nutricional. Dessa forma, o material servirá de suporte aos profissionais e para que os portadores de diabetes superem dúvidas e dificuldades que permeiam o autocuidado referente à alimentação para o controle da doença. 


\section{CONFLITOS DE INTERESSE}

Não há conflitos de interesses a declarar.

\section{CONTRIBUIÇÕES}

Todos os autores contribuíram com a elaboração e delineamento do estudo; a aquisição, análise e interpretação dos dados; a redação e/ou revisão do manuscrito.

\section{REFERÊNCIAS}

1. Sociedade Brasileira de Diabetes. Diretrizes da Sociedade Brasileira de Diabetes 2017-2018. São Paulo: Editora Clannad; 2017.

2. International Diabetes Federation. IDF Diabetes Atlas [Internet]. $8^{a}$ ed. Brussels: International Diabetes Federation; 2017.

3. Rossaneis MA, Haddad MCFL, Mathias TAF, Marcon SS. Diferenças entre mulheres e homens diabéticos no autocuidado com os pés e estilo de vida. Rev Latinoam Enferm [Internet]. 2016 [acesso em 2018 Ago 30];24:e2761. Disponível em: http://www.scielo.br/scielo.php?script=sci_arttext\&pid=S0104$11692016000100384 \& \operatorname{lng}=$ en

4. Ministério da Saúde (BR). Secretaria de Vigilância à Saúde. Secretaria de Atenção à Saúde. Política Nacional de Promoção da Saúde: PNaPS: revisão da Portaria MS/GM nº 687, de 30 de março de 2006. Brasília: Ministério da Saúde; 2014.

5. Ministério da Saúde (BR). Secretaria de Atenção à Saúde. Departamento de Atenção Básica. Diretrizes para o cuidado das pessoas com doenças crônicas nas redes de atenção à saúde e nas linhas de cuidado prioritárias. Brasília: Ministério da Saúde; 2013.

6. Janini JP, Bessler D, Vargas AB. Educação em saúde e promoção da saúde: impacto na qualidade de vida do idoso. Saúde Debate [Internet]. 2015 [acesso em 2019 Mar 02];39(105):480-90. Disponível em: http://www. scielo.br/pdf/sdeb/v39n105/0103-1104-sdeb-39-105-00480.pdf

7. Estrela KCA, Alves ACC, Gomes TT, Isosaki M. Adesão às orientações nutricionais: uma revisão de literatura. DEMETRA: Alimentação, Nutrição \& Saúde [Internet]. 2017 [acesso em 2018 Ago 15];12(1):249-74. Disponível em: https://www.e-publicacoes.uerj.br/index.php/demetra/article/view/22407/21344

8. Costa JA, Balga RSM, Alfenas RCG, Cotta RMM. Promoção da saúde e diabetes: discutindo a adesão e a motivação de indivíduos diabéticos participantes de programas de saúde. Ciênc Saúde Colet [Internet]. 2011 [acesso em 2018 Ago 15];16(3):2001-9. Disponível em: http://www.scielo.br/scielo.php?script=sci_ arttext\&pid=S1413-81232011000300034\&lng=en

9. Benevides JL, Coutinho JFV, Pascoal LC, Joventino ES, Martins MC, Gubert FA, et al. Construção e validação de tecnologia educativa sobre cuidados com úlcera venosa. Rev Esc Enferm USP [Internet]. 2016 [acesso em 2018 Ago 15];50(2):309-16. Disponível em: http://www.scielo.br/pdf/reeusp/v50n2/pt_0080-6234reeusp-50-02-0309.pdf

10. Franco RC. Avaliação do Manual de orientação nutricional para pessoas com diabetes mellitus tipo 2 [tese]. São Paulo: Universidade de São Paulo; 2015.

11. Oliveira SC, Lopes MVO, Fernandes AFC. Construção e validação de cartilha educativa para alimentação saudável durante a gravidez. Rev Latinoam Enferm [Internet]. 2014 [acesso em 2018 Ago 16];22(4):611-20. Disponível em: http://www.scielo.br/pdf/rlae/v22n4/pt_0104-1169-rlae-22-04-00611.pdf

12. Alexandre NMC, Coluci MZO. Validade de conteúdo nos processos de construção e adaptação de instrumentos de medidas. Ciênc Saúde Colet. 2011;16(7):3061-8.

13. Polit DF, Beck CT. Fundamentos da pesquisa em enfermagem: avaliação de evidências para a prática de enfermagem. $7^{\text {a }}$ ed. Porto Alegre: ArtMed; 2011. 
14. Echer IC. The development of handbooks of health care guidelines. Rev Latinoam Enferm. 2005;13(5):754-7.

15. Ministério da Saúde (BR). Secretaria de Atenção à Saúde. Departamento de Atenção Básica. Guia alimentar para a população brasileira. $2^{\mathrm{a}}$ ed. Brasília: Ministério da Saúde; 2014.

16. Sociedade Brasileira de Diabetes. Departamento de Nutrição e Metabologia da SBD. Manual de Nutrição: pessoa com diabetes 2009. São Paulo: SBD; 2009.

17. American Diabetes Association. Standards of Medical Care in Diabetes - 2017. Diabetes Care. 2017;40(Suppl 1).

18. Moreira MF, Nóbrega MML, Silva MIT. Comunicação escrita: contribuição para a elaboração de material educativo em saúde. Rev Bras Enferm [Internet]. 2003 [acesso em 2018 Set 03];56(2):184-8. Disponível em: http://www.scielo.br/scielo.php?script=sci_arttext\&pid=S0034-71672003000200015\&lng=en

19. Joventino ES. Desenvolvimento de escala para mensurar a autoeficácia materna na prevenção da diarreia infantil [dissertação]. Fortaleza: Universidade Federal do Ceará; 2010.

20. Fehring RJ. The Fehring Model. In: Carrol-Johnson RM, Paquete M, editores. Classification of nursing diagnoses: proceedings of the Tenth Conference. Philadelphia: JB Lippincott; 1994. p. 55-62.

21. Maniva SJCF. Elaboração e validação de tecnologia educativa sobre acidente vascular cerebral para prevenção da recorrência [tese]. Fortaleza: Universidade Federal do Ceará; 2016.

22. Lynn MR. Determination and quantification of content validity. Nurs Res. 1986;35(9):382-5.

23. Polit DF, Beck CT. The content validity index: are you sure you know what's being reported? Critique and recommendations. Res Nurs Health [Internet]. 2006 [acesso em 2018 Set 03];29(5):489-97. Disponível em: https://pdfs.semanticscholar.org/537d/5a0f09968979b4cf4e8b0213a8f39257b393.pdf

24. Ministério da Saúde (BR). Plano de ações estratégicas para o enfrentamento das Doenças Crônicas Não Transmissíveis (DCNT) no Brasil 2011-2022. Brasília: Ministério da Saúde; 2011.

25. Câmara C. Mapeamento político da saúde no Brasil com foco em diabetes e doenças cardiovasculares. São Paulo: Sociedade Brasileira de Diabetes; 2018.

26. Brasil. Projeto de Lei da Câmara n. ${ }^{\circ 133}$, de 2017. Institui a Política Nacional de Prevenção do Diabetes e de Assistência Integral à Saúde da Pessoa Diabética [Internet]. 2019 [acesso em 2019 Jan 02]. Disponível em: https://www25.senado.leg.br/web/atividade/materias/-/materia/131364

27. Silveira MS, Cogo ALP. Contribuições das tecnologias educacionais digitais no ensino de habilidades de enfermagem: revisão integrativa. Rev Gaúcha Enferm [Internet]. 2017 [acesso em 2019 Abr 19];38(2):e66204. Disponível em: http://www.revenf.bvs.br/scielo.php?script=sci_arttext\&pid=S1983$14472017000200501 \&$ Ing $=p t$

28. Ramos LMH, Araújo RFR. Uso de cartilha educacional sobre diabetes mellitus no processo de ensino e aprendizagem. Ensino, Saúde e Ambiente [Internet]. 2017 [acesso em 2019 Abr 02];10(3):94-105. Disponível em: http://periodicos.uff.br/ensinosaudeambiente_backup/article/view/14771/9372

29. Moura IH, Silva AFR, Rocha AESH, Lima LHO, Moreira TMM, Silva ARV. Construção e validação de material educativo para prevenção de síndrome metabólica em adolescentes. Rev Latinoam Enferm [Internet]. 2017 [acesso em 2018 Set 05];25:e2934. Disponível em: http://www.scielo.br/scielo.php?script=sci_ arttext\&pid=S0104-11692017000100383\&Ing=pt

30. Cruz FOAM, Ferreira EB, Vasques CI, Mata LFR, Reis PED. Validação de manual educativo para pacientes com câncer de cabeça e pescoço submetidos à radioterapia. Rev Latinoam Enferm [Internet]. 2016 [acesso em 2018 Set 04];24:e2706. Disponível em: http://www.scielo.br/scielo.php?script=sci_arttext\&pid=S0104$11692016000100337 \&$ Ing=en

31. Mendonça SCB, Zanetti ML, Sawada NO, Barreto IDC, Andrade JS, Otero LM. Construção e validação do Instrumento Avaliação do Autocuidado para pacientes com diabetes mellitus tipo 2. Rev Latinoam Enferm [Internet]. 2017 [acesso em 2018 Set 06];25:e2890. Disponível em: http://www.scielo.br/pdf/rlae/v25/pt_01041169-rlae-25-e2890.pdf 
32. Teles LMR, Oliveira AS, Campos FC, Lima TM, Costa CC, Gomes LFS, et al. Construção e validação de manual educativo para acompanhantes durante o trabalho de parto. Rev Esc Enferm USP. 2014;48(6):97784.

33. Lima ACMACC, Bezerra KC, Sousa DMN, Rocha JF, Oriá MOB. Construção e validação de cartilha para prevenção de transmissão vertical do HIV. Acta Paul Enferm. 2017;30(2):181-9.

\section{Endereço para correspondência:}

Marília de Sousa Gonçalves

Universidade Federal do Ceará - UFC

Rua Cel. Nunes de Melo, s/n

Bairro: Rodolfo Teófilo

CEP: 60430-270 - Fortaleza - CE - Brasil

E-mail: marilia-s-goncalves@hotmail.com

Como citar: Gonçalves MS, Celedônio RF, Targino MB, Albuquerque TO, Flauzino PA, Bezerra AN, et al. Construção e validação de cartilha educativa para promoção da alimentação saudável entre pacientes diabéticos. Rev Bras Promoç Saúde. 2019;32:7781. 